\title{
Reactive Lymphoid Hyperplasia of the liver in a patient with colon
} cancer: report of two cases

\author{
Hiroki Takahashi*1, Hirozumi Sawai ${ }^{1}$, Yoichi Matsuo ${ }^{1}$, Hitoshi Funahashi ${ }^{1}$, \\ Mikinori Satoh ${ }^{1}$, Yuji Okada ${ }^{1}$, Hiroshi Inagaki ${ }^{2}$, Hiromitsu Takeyama ${ }^{1}$ and \\ Tadao Manabe ${ }^{1}$
}

Address: ${ }^{1}$ Department of Gastroenterological Surgery, Nagoya City University Graduate School of Medical Sciences, Kawasumi 1, Mizuho-cho, Mizuho-ku, Nagoya, Japan and 2Department of Pathology, Nagoya City University Graduate School of Medical Sciences, Kawasumi 1, Mizuhocho, Mizuho-ku, Nagoya, Japan

Email: Hiroki Takahashi* - takahasi@med.nagoya-cu.ac.jp; Hirozumi Sawai - sawai@med.nagoya-cu.ac.jp;

Yoichi Matsuo - matsuo@med.nagoya-cu.ac.jp; Hitoshi Funahashi - funa84@med.nagoya-cu.ac.jp; Mikinori Satoh - miki@med.nagoya-cu.ac.jp; Yuji Okada - yuji@med.nagoya-cu.ac.jp; Hiroshi Inagaki - hinagaki@med.nagoya-cu.ac.jp; Hiromitsu Takeyama - takeyama@med.nagoyacu.ac.jp; Tadao Manabe - mnb@med.nagoya-cu.ac.jp

* Corresponding author

Published: 12 September 2006

BMC Gastroenterology 2006, 6:25 doi:10.1 186/I47/-230X-6-25
Received: 16 June 2006

Accepted: 12 September 2006

This article is available from: http://www.biomedcentral.com/I47I-230X/6/25

(C) 2006 Takahashi et al; licensee BioMed Central Ltd.

This is an Open Access article distributed under the terms of the Creative Commons Attribution License (http://creativecommons.org/licenses/by/2.0), which permits unrestricted use, distribution, and reproduction in any medium, provided the original work is properly cited.

\section{Abstract}

Background: Reactive lymphoid hyperplasia (RLH) of the liver is very rarely reported, and we encountered two cases of RLH of the liver in a patient with colon cancer.

Case presentation: In the first case, a 77-year-old woman was admitted for the surgical removal of a ascending colon cancer. A hepatic tumor in the left lobe was concurrently revealed by computed tomography (CT), and magnetic resonance imaging (MRI). The appearance suggested liver metastasis. Right hemicolectomy and partial hepatectomy were performed. On histopathological examination, lymphoid follicles with germinal centers were seen in the tumor-like lesion, and remarkable lymphoid infiltration with germinal centers was seen in the portal area around the nodule. Immunohistochemical studies revealed polyclonality of infiltrating lymphocyte. Consequently, this nodular lesion was diagnosed as RLH of the liver. In the second case, a 64-yearold woman who had a radical right hemicolectomy for stage II ascending colon cancer 10 years ago was admitted with dysuria. A hepatic tumor in the left lobe was concurrently revealed by $C T$ and MRI, suggesting hepatocellular carcinoma. A left lateral segmentectomy was performed. Microscopically, this lesion revealed the almost same findings as the first case, so this nodular lesion was diagnosed as RLH of the liver.

Conclusion: Our two cases were the first report of RLH of the liver accompanying colon cancer. Because there are a very few cases, so it is not clear whether the malignancies were involved in the onset of RLH. But we believe that new factors involved in the onset mechanism of RLH may be identified by carefully monitoring the clinical course of our two patients. 


\section{Background}

Reactive lymphoid hyperplasia (RLH) is reported to occur in the gastrointestinal tract [1], skin [2], lung [3], thyroid [4], orbit [5], and pancreas [6]; however, it is very rarely reported in the liver. To the best of our knowledge, only 12 cases with RLH of the liver have so far been reported in the English literature [7-15]. RLH is thought to represent an immune-mediated reactive phenomenon, and very few cases may arise in association with malignant tumor. But, there are no reports mentioned about the relationship between RLH and malignant tumor. Our two cases were the first report of RLH of the liver accompanying colon cancer. So we report two cases of RLH of the liver accompanying colon cancer and discuss its morphological features.

\section{Case presentation Case I}

A 77-year-old woman was admitted to our hospital with right upper abdominal pain in December 2004. Barium enema and colonoscopy revealed an ulcerative tumor in the ascending colon, which was diagnosed as moderately differentiated adenocarcinoma on biopsy. Physical examination showed no remarkable abnormalities. Neither hepatomegaly nor splenomegaly were apparent. Serum hepatitis B surface antigen and hepatitis $\mathrm{C}$ antibody were negative, and she had no history of alcohol intake or blood transfusion. Laboratory data on admission including liver function tests were unremarkable. Carcinoembryonic antigen (CEA), carbohydrate antigen 19-9 (CA199 ), and $\alpha$-fetoprotein (AFP) were within normal ranges. Abdominal ultrasonography showed a hypoechoic, 1.5$\mathrm{cm}$ diameter mass in the left hepatic lobe (S3). Computed tomography (CT) revealed a low-density area in the same area, the interior of which enhanced equally. The outline of the mass was particularly enhanced in equilibrium phase (Fig. 1). Magnetic resonance imaging (MRI) demonstrated a slightly low-intensity lesion on T1-weighted images; this was high-Intensity on T2-weighted images (Fig. 2). Furthermore, superparamagnetic iron oxide (SPIO) uptake was not detected on T2-weighted images. Consequently, as metastatic liver tumor could not be definitively excluded, the patient underwent right hemicolectomy and S3 partial hepatectomy on 1 November 2004.

An ulcerated tumor with sharply demarcation was present in the ascending colon and histopathological examination revealed moderately differentiated adenocarcinoma. No lymph node metastasis was detected. Macroscopically, cut section of the resected liver showed a well-circumscribed, encapsulated, yellowish-white mass in S3 of the liver. It measured $1.5 \mathrm{~cm}$ in diameter (Fig. 3). The tumor appeared relatively soft and elastic and was composed of many lymphoid follicles with large germinal centers con-
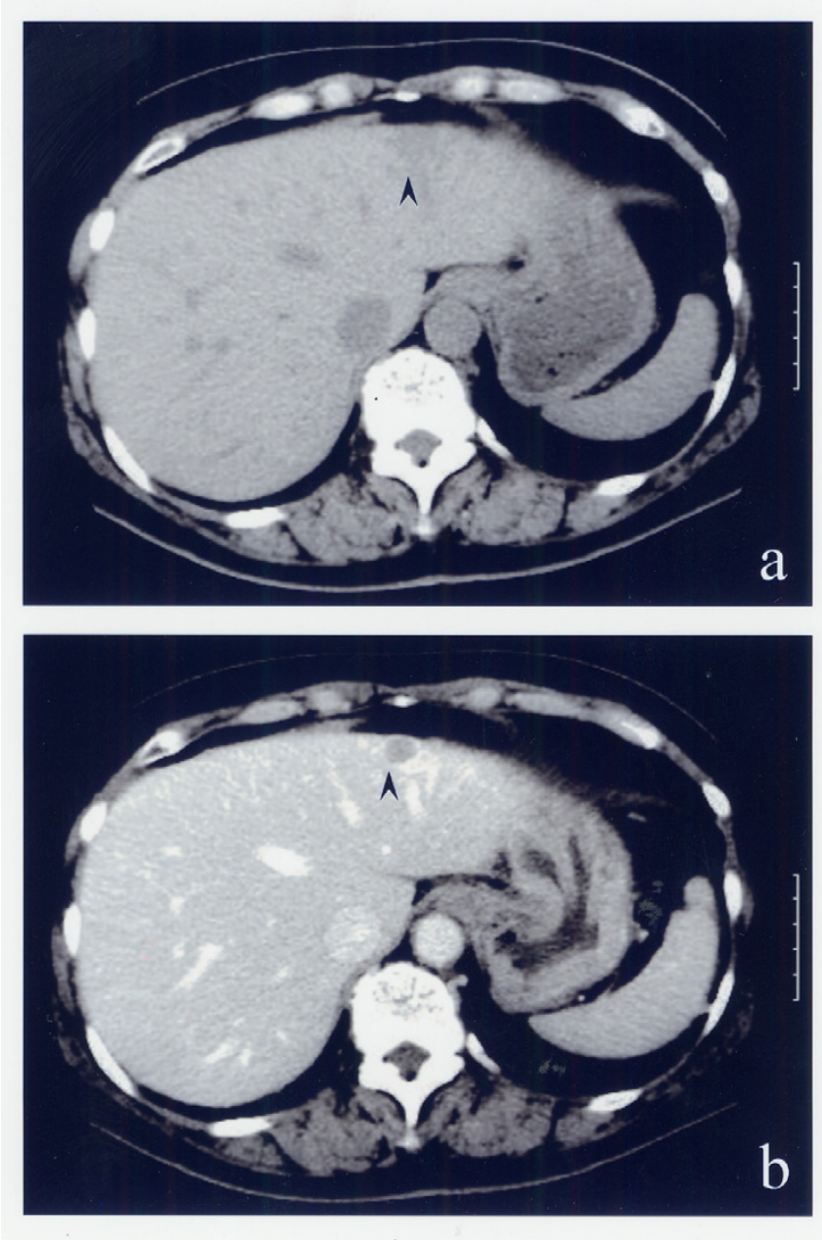

\section{Figure I}

a) Abdominal CT shows the $1.5-\mathrm{cm}$ diameter low-density mass in size in the S3. b) This lesion was slightly enhanced, and its periphery was noticeably enhanced on equilibrium phase.

taining small polymorphic lymphocytes (Fig. 4). Many plasma cells surrounded mature lymphoid aggregates. Furthermore, remarkable lymphoid infiltration with germinal center formation was seen in the portal area around the nodule. However, the hepatic parenchyma distant from this nodule was normal and lymphoid infiltration was not detected in the portal area. No evidence of adenocarcinoma metastasis and no cytologic atypia of the germinal centers or interfollicular areas were detected. Immunohistochemical studies revealed the germinal centers to be composed of CD-20-positive and Bcl-2-negative lymphocytes. Furthermore, lymphocytes surrounding the germinal centers and interfollicular areas were positive for CD-3 and Bcl-2. Plasma cells were positive for both $\kappa$ and $\lambda$ light chains at an equal frequency (data not shown). These findings revealed the polyclonal origins of the infiltrating lymphocytes. Consequently, this nodular 

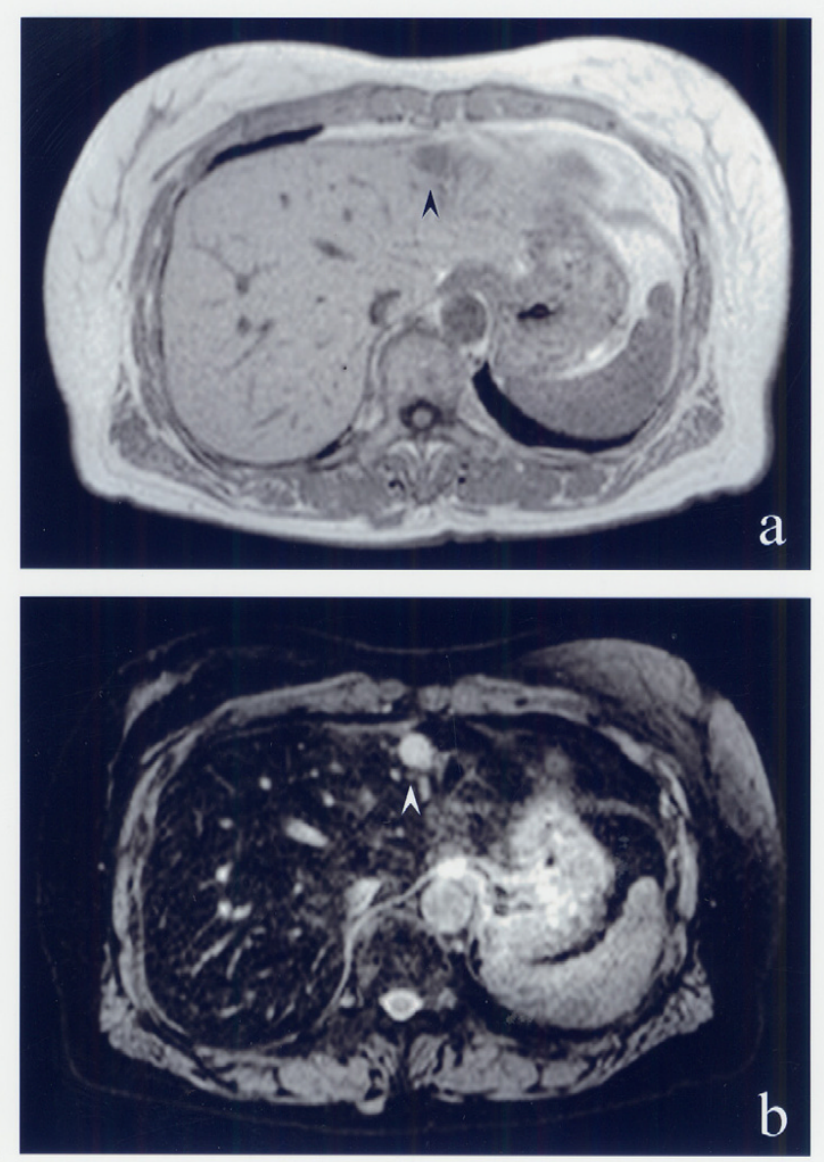

\section{Figure 2}

a) MRI showed a low-intensity lesion on the TI-weighted image. b) The border of the lesion was clearly visualized on the T2-weighted image with SPIO.

lesion was diagnosed as RLH of the liver. No recurrence has occurred during 20 months of postoperative follow up.

\section{Case 2}

A 64-year-old woman who had a radical right hemicolectomy for stage II ascending colon cancer 10 years ago was admitted to our hospital with dysuria in July 2005. A hepatic tumor in the left lobe of her liver was concurrently revealed by CT scan. Serum hepatitis B surface antigen and hepatitis $\mathrm{C}$ antibody were negative, and she had no history of alcohol intake or blood transfusion. Laboratory data on admission including liver function tests were unremarkable. CEA, CA19-9, and AFP were within normal ranges. Abdominal ultrasonography showed a hypoechoic, $9 \times 7 \mathrm{~mm}$ diameter mass in the left hepatic lobe

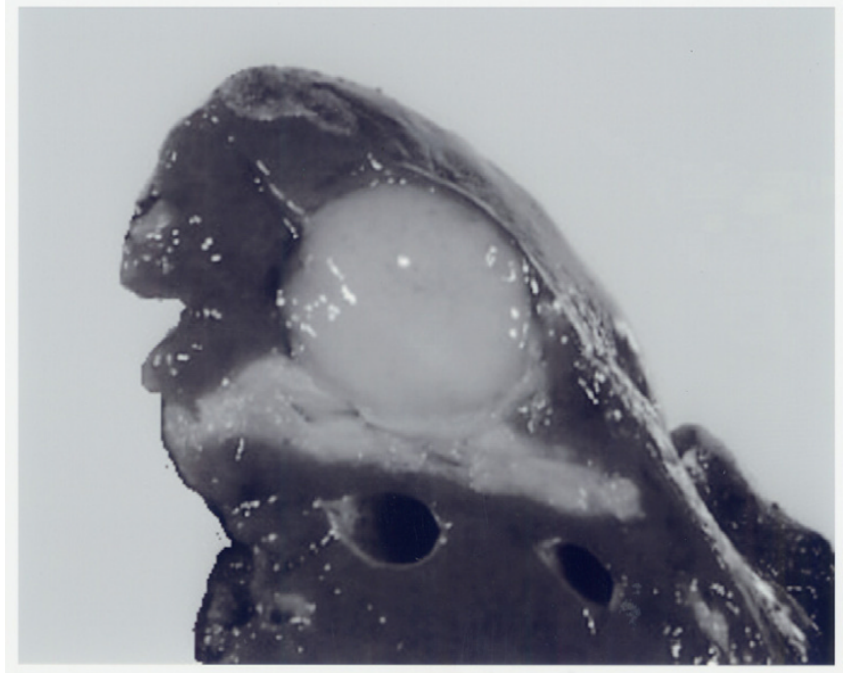

Figure 3

A cut section of the resected liver showed a well-circumscribed, yellowish-white mass in S3.

(S2). Plain CT examination revealed a low-density area, the mass was enhanced by the contrast medium in early phase. At a late phase, the mass was showed as a low-density area. MRI demonstrated a slightly low-intensity lesion on T1-weighted images and a high-Intensity on T2weighted images. SPIO uptake was not detected on T2weighted images. At an early phase, Dynamic MRI revealed low-intensity mass in S2 (Fig. 5a). At a late phase, the mass was showed as a low-intensity area (Fig. 5b). Consequently, this nodular lesion was diagnosed hepatocellular carcinoma rather than liver metastasis, the patient was carried out left lateral segmentectomy on 7 September 2005.

Macroscopically, the resected liver had smooth surface and a normal appearance, cut section of the resected liver showed a well-circumscribed, not encapsulated, whitish mass in S2 of the liver (Fig. 6). It measured $1.0 \mathrm{~cm}$ in diameter. Microscopically, this lesion revealed the almost same findings as Case 1, so this nodular lesion was diagnosed as RLH of the liver, too. No recurrence has occurred during 10 months of postoperative follow up.

\section{Discussion}

Reactive lymphoid hyperplasia is recognized as a region that is well demarcated and characterized by hyperplastic lymphoid follicles with reactive germinal centers, which consist of polymorphic and polyclonal cell populations and aggregations of mature lymphocytes, plasma cells, and other types of inflammatory cells [12]. In the liver, these reactive lesions have been variously termed RLH, nodular lymphoid lesions [14], and pseudolymphoma. 

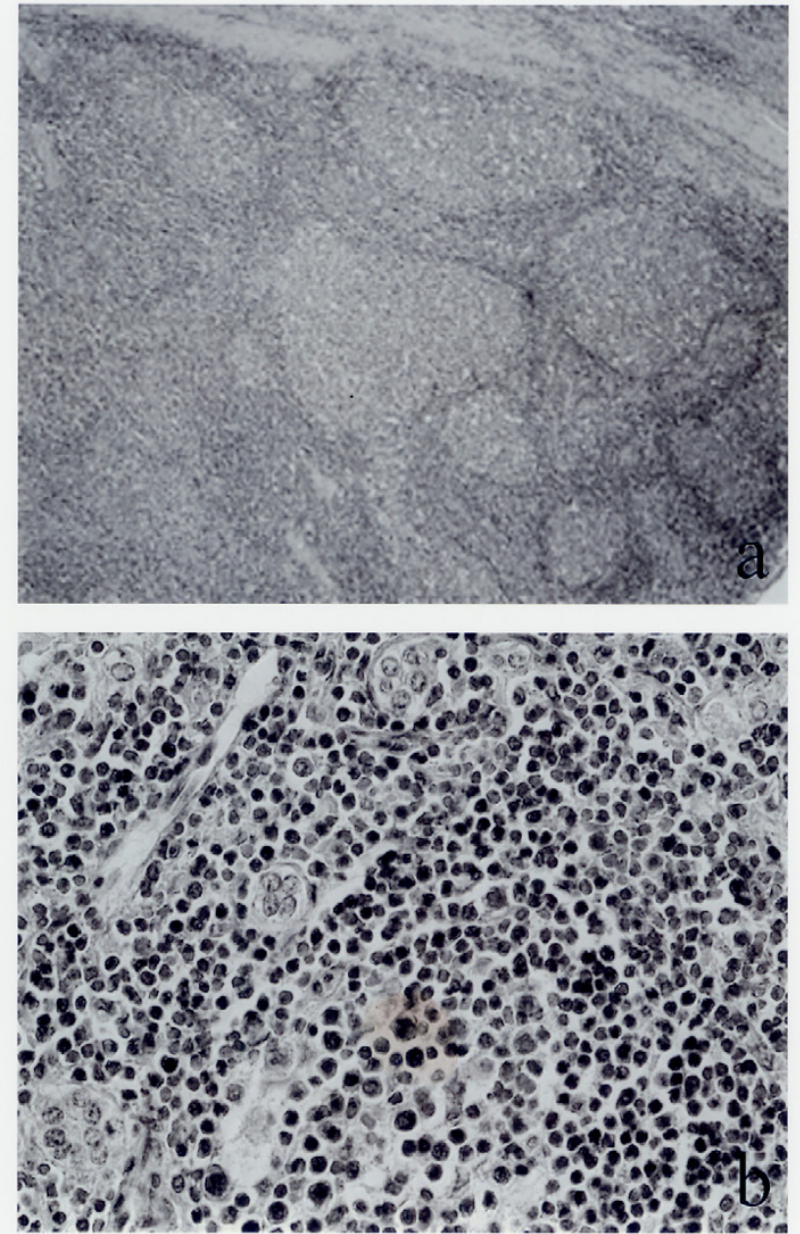

\section{Figure 4}

Many lymphoid follicles with germinal centers were seen in the tumor-like hepatic lesion ( $\mathrm{a}: \times 40, \mathrm{~b}: \times 400)$, HE staining.

These lesions are very rare, and they appear clinically to indicate the same disease. We decided to use the term "RLH of the liver" because this most closely reflects the pathological features of the disease. However, the term "pseudolymphoma" is problematic because it indicates a low-grade type of MALT lymphoma. Since malignant transformation is reported in this type of MALT lymphoma, surgical resection is indicated. Therefore, even when pseudolymphoma is diagnosed preoperatively, some patients have undergone resective surgery [13]. In contrast, as RLH is not considered to have malignant potential, therapeutic strategies are totally different from those for low-grade MALT-type lymphoma. We therefore believe it is necessary to eliminate the ambiguous term "pseudolymphoma" and clearly differentiate RLH from low-grade MALT-type lymphoma. Some past studies have also advocated this approach to classification, and Sharifi

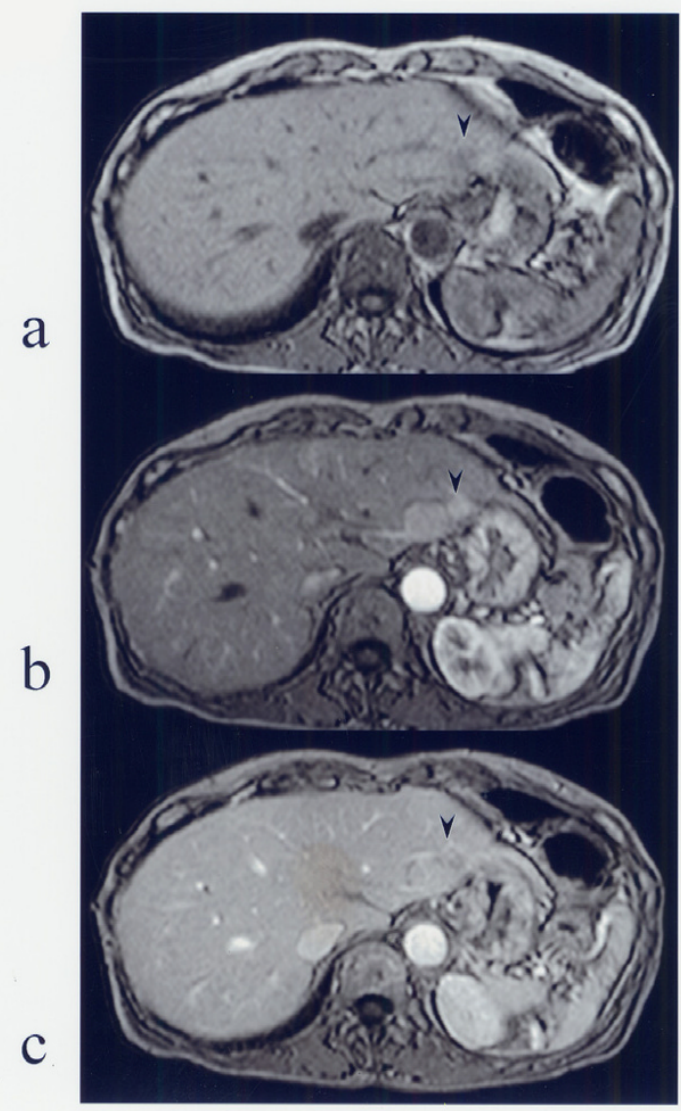

\section{Figure 5}

Dynamic MRI. a) Plain MRI showed the I.0-cm diameter lowintensity mass in size in the S2. b) This nodular lesion was enhanced at an early phase. c) At a late phase, this lesion was showed as low-intensity mass than surrounding liver tissue.

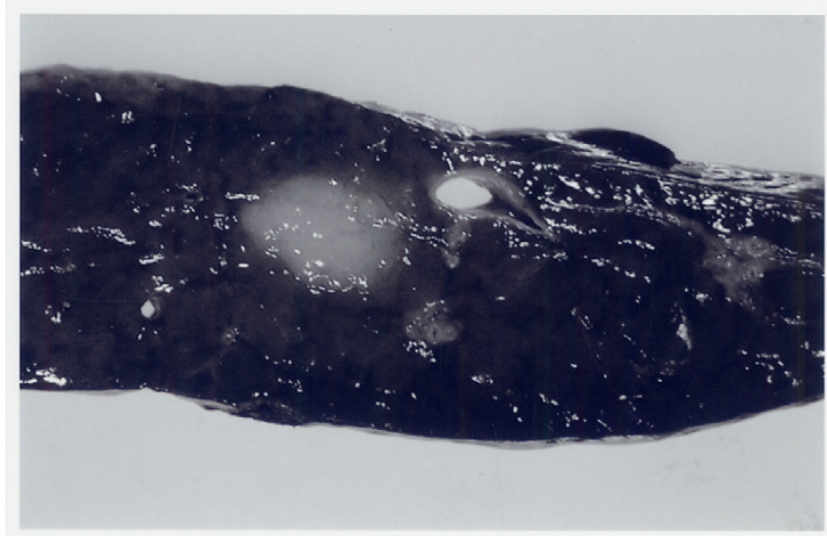

Figure 6

A cut section of the resected liver showed a well-circumscribed, whitish mass in S2. 


\begin{tabular}{|c|c|c|c|c|c|c|c|c|c|c|}
\hline No. & age & sex & segment & Size $(\mathrm{cm})$ & $\mathrm{CT}$ & MRI & US & Angio & association & Author/Year \\
\hline 1 & 69 & $\mathrm{~F}$ & Rt post lobe & $1.7 * 1.0 * 0.9$ & $\begin{array}{c}\text { plain: low } \\
\text { not enhanced }\end{array}$ & - & - & - & 7 yeas after RCC ope. & Pantanowitz/200I \\
\hline 2 & 47 & $\mathrm{~F}$ & S7 & 1.7 & $\begin{array}{l}\text { plain: low } \\
\text { enhanced }\end{array}$ & $\begin{array}{l}\text { TI- low } \\
\text { T2-high }\end{array}$ & $\begin{array}{l}\text { hypoechoic } \\
\text { hyperechoic rim }\end{array}$ & hypervascular & Hashimoto's thyroiditis & Nagano/I999 \\
\hline 3 & 67 & $\mathrm{~F}$ & Lt lobe & 2 & $\begin{array}{l}\text { plain: low } \\
\text { sl. enhanced }\end{array}$ & - & hypoechoic & hypervascular & - & Tanizawa//996 \\
\hline 4 & 52 & $\mathrm{~F}$ & Rt lobe & 0.4 & - & - & - & - & primaly biliary cirrhousis & Sharifi//999 \\
\hline 5 & 56 & $\mathrm{~F}$ & Lt lobe & 1.5 & - & - & - & - & $\begin{array}{l}\text { primaly biliary cirrhousis } \\
\text { CREST synd. }\end{array}$ & Sharifi//999 \\
\hline 6 & 56 & $M$ & - & 0.7 & - & - & - & - & chronic diverticular & Sharifi//999 \\
\hline 7 & 15 & $\mathrm{~F}$ & - & - & - & - & - & - & primary immunodeficiency synd. & Snover/198I \\
\hline 8 & 49 & $\mathrm{~F}$ & Rt post lobe & 2 & Tumor lesion $(+)$ & $\begin{array}{l}\text { TI-low } \\
\text { T2-high }\end{array}$ & tumor lesion $(+)$ & hypervascular & Sjögren's synd. & Okubo/200I \\
\hline 9 & 42 & $\mathrm{~F}$ & S6, S3 & $\begin{array}{l}\text { S3:1.1 } \\
\text { S6:1.6 }\end{array}$ & $\begin{array}{l}\text { low density } \\
\text { Enhance-CT:iso }\end{array}$ & - & hypoechoic & tumor stain $(+)$ & chronic hepatitis B, IFN- $\alpha$ & Ohtsu/l994 \\
\hline 10 & 72 & M & S3 & $2.1 * 1.4$ & $\begin{array}{l}\text { Plain: no tumor } \\
\text { Early: high, late: iso-low }\end{array}$ & $\begin{array}{l}\text { TI-low } \\
\text { T2-high }\end{array}$ & hypoechoic & failure & $\begin{array}{l}\text { chronic hepatitis } C \\
\text { early gastric cancer }\end{array}$ & $\mathrm{Kim} / \mathrm{I} 997$ \\
\hline 11 & 66 & $\mathrm{~F}$ & Rt lobe & $1.0 * 1.0$ & $\begin{array}{c}\text { plain: low } \\
\text { not enhanced }\end{array}$ & - & hypoechoic & tumor stain $(+)$ & DM & Katayanagi// 994 \\
\hline 12 & 85 & $\mathrm{~F}$ & Rt lobe & $\begin{array}{l}1.4 * 0.8 * 1.0 \\
0.7 * 0.8 * 0.7\end{array}$ & - & - & - & - & gastric cancer & Grouls/l984 \\
\hline \multicolumn{11}{|c|}{ Our cases } \\
\hline 1 & 77 & $\mathrm{~F}$ & S3 & 1.5 & plain: low, enhance sl.(+) & $\begin{array}{l}\text { TI-low } \\
\text { T2-high }\end{array}$ & hypoechoic & - & colon cancer & \\
\hline 2 & 64 & $\mathrm{~F}$ & S2 & $0.9 * 0.7$ & $\begin{array}{l}\text { Plain: low, enhance-CT } \\
\text { Early:high, late:low }\end{array}$ & $\begin{array}{l}\text { TI-low } \\
\text { T2-high }\end{array}$ & hypoechoic & tumor stain(-) & 10 years after colon cancer & \\
\hline
\end{tabular}


and colleagues [14] have proposed clear diagnostic criteria. Immunohistological and genetic tests to ascertain whether infiltrating lymphocytes are monoclonal or polyclonal enable differentiation of RLH from low-grade MALT-type lymphoma.

By previously reported cases, in terms of imaging findings, RLH of the liver is depicted as a hypoechoic lesion on ultrasound and as a low-density lesion on plain CT, but contrast CT findings vary. Both MRI T1-low and T2-high intensity images yield the same results, and angiography shows hypervascularity. In our patients, results of preoperative diagnostic imaging conformed to this pattern; i.e. low-echo and plain CT demonstrated a low-density lesion, while contrast CT showed mild enhancement. Moreover, on equilibrium phase of contrast CT in the first patient, the area around RLH was depicted as a high-density area, possibly indicating a capsule. The present study was the first to conduct contrast SPIO-enhanced MRI in hepatic RLH. No SPIO uptake into the mass occurred, and the boundary with the normal liver was very clear. The further discussion of such rare cases based on the imaging findings including SPIO-enhanced MRI will provide more information about this rare entity. But, at this point in time, it is very difficult to completely rule out malignancies such as HCC, metastatic liver tumors, or malignant lymphomas based solely on diagnostic imaging findings. Ultimately, preoperative biopsy is therefore an essential diagnostic tool.

While the cause of RLH has not been clarified, most patients are middle-aged or elderly women. In terms of underlying diseases, various chronic inflammatory diseases, such as chronic hepatitis, Sjogren's syndrome, chronic thyroiditis, and primary biliary cirrhosis, have been confirmed. Hence, autoimmune mechanisms are most likely involved. In our cases, the patients were a middle-aged woman and lymphoid infiltration was observed in the portal area around the nodule, suggesting a similar mechanism. However, lymphoid infiltration was confined to the area around the nodule, the rest of the hepatic parenchyma was normal, and hepatitis virus markers were negative, suggesting that this lesion was local rather than diffuse. Furthermore, as systemic immunologic abnormalities were not detected, our cases did not appear to be associated with systemic autoimmune disease. Including the present patients, five cases of hepatic RLH accompanying malignancies have been reported $[8,11,15]$, some of which had asynchronous onset, but the present study is the first to report RLH of the liver accompanying colon cancer. Because there are a very few cases, so it is not clear whether the malignancies were involved in the onset of RLH. Furthermore, characteristic imaging findings or morphological features in these patients were not detected. But we believe that new factors involved in the onset mechanism of RLH may be identified by carefully monitoring the clinical course of our two patients.

\section{Conclusion}

In summary, we report two cases of RLH of the liver in a patient with colon cancer. RLH of the liver is extremely rare and it is very difficult to think of RLH of the liver as differential diagnosis.

\section{Abbreviations}

RLH: Reactive lymphoid hyperplasia, MALT: Mucosaassociated lymphoid tissue, US: ultrasonography, CT: Computed tomography, MRI: Magnetic resonance imaging, SPIO: Superparamagnetic iron oxide

\section{Competing interests}

The author(s) declare that they have no competing interests.

\section{Authors' contributions}

HT, MS, YO, and HT carried out the clinical examination and operation. HT, HF, HS, and HI performed pathological analysis. HT and YM participated in the design of the study. TM conceived of the study, and participated in its design and coordination. All authors read and approved the final manuscript.

\section{Acknowledgements}

Written consent was obtained from the patients for publication of study.

\section{References}

I. Torigian DA, Levine MS, Gill NS, Rubesin SE, Fogt F, Schultz CF, Furth $E E$, Laufer I: Lymphoid hyperplasia of the stomach: radiographic findings in five adult patients. Am J Roentgenol 200I, 177:7|-75.

2. Arai E, Shimizu M, Hirose T: A review of $\mathbf{5 5}$ cases of cutaneous lymphoid hyperplasia: reassessment of the histopathologic findings leading to reclassification of 4 lesions as cutaneous marginal zone lymphoma and 19 as pseudolymphomatous folliculitis. Hum Pathol 2005, 36:505-II.

3. Abbondanzo SL, Rush W, Bijwaard KE, Koss MN: Nodular lymphoid hyperplasia of the lung: a clinicopathologic study of 14 cases. Am J Surg Pathol 2000, 24:587-97.

4. Mizukami Y, Nonomura A, Michigishi T, Noguchi M, Nakamura S, Ishizaki T: Pseudolymphoma of the thyroid gland. A case report. Pathol Res Pract 1996, 192:166-9.

5. Westacott S, Garner A, Moseley IF, Wright JE: Orbital lymphoma versus reactive lymphoid hyperplasia: an analysis of the use of computed tomography in differential diagnosis. $\mathrm{Br} J \mathrm{Oph}$ thalmol 1991, 75:722-5.

6. Nakashiro $H$, Tokunaga $O$, Watanabe $T$, Ishibashi $K$, Kuwaki $T$ : Localized lymphoid hyperplasia (pseudolymphoma) of the pancreas presenting with obstructive jaundice. Hum Pathol |99|, 22:724-6.

7. Snover DC, Filipovich AH, Dehner LP, Krivit W: Pseudolymphoma. A case associated with primary immunodeficiency disease and polyglandular failure syndrome. Arch Pathol Lab Med 198I, 105:46-9.

8. Grous V: Pseudolymphoma (inflammatory pseudotumor) of the liver. Zentralbl Allg Pathol 1987, I33:565-8.

9. Katayanagi K, Terada T, Nakanuma Y, Ueno T: A case of pseudolymphoma of the liver. Patho Inter 1994, 44:704-II.

10. Okubo H, Maegawa H, Ogawa K, Wada R, Sekigawa I, lida N, Maekawa T, Hashimoto H, Sato N: Pseudolymphoma of the liver 
associated with Sjögren's syndrome. Scand J Rheumatol 200I, 30:117-9.

II. Kim SO, Hayashi Y, Kang KB, Soe CG, Kim JH, Yang MK, Itoh H: A case of pseudolymphoma of the liver with chronic hepatitis C. J Hepatol 1997, 26:209-14.

12. Nagano K, Fukuda Y, Nakano I, Katano Y, Toyoda H, Nonami T, Nagasaka T, Hayakawa T: Case report: Reactive lymphoid hyperplasia of liver coexisting with chronic thyroiditis: Radiographical characteristics of the disorder. J Gastroentrol Hepatol 1999, 14:163-67.

13. Shiozawa K, Kinoshita H, Tsuruta H, Nakazawa K, Naitou S, Koga M, Takeshima F, Omagari K, Mizuta Y, Murata I, Kohno S: A case of pseudolymphoma of the liver diagnosed before operation. Nipponn Syoukakibyou Gakkai Zassi 2004, I 01 1:772-78.

14. Sharifi S, Murphy M, Loda M, Pinkus GS, Khettry U: Nodular lymphoid lesion of the liver. An immune-mediated disorder mimicking low-grade malignant lymphoma. Am J Surg Patho 1999, 23:302-08.

15. Pantanowitz L, Saldinger PF, Kadin ME: Pathologic Quiz Case: Hepatic mass in a patient with renal cell carcinoma. Arch Pathol Lab Med 200I, I 25:577-78.

\section{Pre-publication history}

The pre-publication history for this paper can be accessed here:

http://www.biomedcentral.com/1471-230X/6/25/pre pub

Publish with Bio Med Central and every scientist can read your work free of charge

"BioMed Central will be the most significant development for disseminating the results of biomedical research in our lifetime. "

Sir Paul Nurse, Cancer Research UK

Your research papers will be:

- available free of charge to the entire biomedical community

- peer reviewed and published immediately upon acceptance

- cited in PubMed and archived on PubMed Central

- yours - you keep the copyright

Submit your manuscript here:

http://www.biomedcentral.com/info/publishing_adv.asp 